ing how the couple due to tidal friction can be transmitted to the core by viscosity, and there would be more difficulty for periods from 0.5 year to 3000 years. Electromagnetic coupling has been suggested, but so far as I know there has been no quantitative examination.
REFERENCES

Jackson, J. I930, $M . N$. 90, 733 .

Jeffreys, H. 1948, $M$. N. 108, 206.

. $1949, M . N$. 109, 670.

Jeffreys, H. and Vicente, R. O. I957a, $M . N$. II7, I42. $1957 \mathrm{~b}$, Ibid., 162.

Poincaré, H. 19ı, Bull. Astr. Paris 26, 32 I.

Takeuchi, H. 1950, Trans. Amer. Geophys. Union 31, $65 \mathrm{I}$.

\title{
LES RELATIONS ENTRE LES MOUVEMENTS DU POLE ET LES FLUCTUATIONS DE LA VITESSE DE ROTATION DE LA TERRE
}

\author{
PAR PAUL MELCHIOR \\ Observatoire Royal de Belgique, Bruxelles, Belgique
}

\begin{abstract}
Résumé. On expose comment on peut tenter une ample synthèse des résultats d'observations astronomiques telles que celles du mouvement du pôle et des fluctuations de la vitesse de rotation de la Terre avec des observations de caractère purement géophysique: les marées terrestres, la circulation atmosphérique générale, le retrait progressif des glaciers, etc. On ne peut espérer donner dès à présent une conclusion à chacun des problèmes soulevés par la confrontation de ces données si diverses et l'on relèvera plusieurs contradictions. L'effort accompli dans le cadre de l'Année Géophysique permettra peut-être de lever certaines d'entre-elles et de parfaire la synthèse évoquée ici.
\end{abstract}

Se proposer l'étude des anomalies que nous révèle l'observation de la rotation de la Terre, c'est aborder un problème de géophysique avec des méthodes d'investigation astronomiques. Qu'il s'agisse de l'étude des composantes du mouvement du pôle, de l'étude des fluctuations de la vitesse de rotation du globe ou de problèmes plus particuliers comme celui de la constante de nutation, l'on devra faire appel à des informations purement géophysiques (séismologie, marées terrestres notamment) si l'on veut progresser dans l'interprétation des phénomènes observés. Géophysiciens et astronomes étudient les mêmes propriétés physiques de la même planète par des voies différentes. Une synthèse riche en perspectives se prépare dans le cadre d'une doctrine commune que par analogie avec l'astronomie géodésique nous appellerions volontiers "astronomie géophysique."

Nous diviserons notre exposé sur les relations entre les mouvements du pôle et les fluctuations de la vitesse de rotation de la Terre en trois parties qui sont trois problèmes essentiellement différents au point de vue du géophysicien mais qui, dans l'observation astronomique, sont étroitement associés:

Premier problème. Relation entre la composante libre du mouvement du pôle, les fluctuations à période semi-mensuelle, mensuelle et semi-annuelle de la vitesse de rotation de la Terre, les marées terrestres, la séismologie, le relèvement isostatique.

Deuxième problème. Relation entre la composante forcée annuelle du mouvement du pôle, la fluctuation à période annuelle de la vitesse de rotation de la Terre, la météorologie et l'océanographie.

Troisième problème. Relation entre le déplacement séculaire du pôle, les variations séculaires de la vitesse de rotation de la Terre, la glaciologie.

I

I. L'étude des phénomènes de marées terrestres et l'étude du mouvement libre du pôle instantané de rotation à la surface de la Terre rentrent dans le cadre d'une seule et même théorie.

En effet, si la Terre était une Terre idéale, indéformable comme le suppose la Mécanique Céleste classique, on pourrait rendre exactement compte par le calcul des caractéristiques de ces deux phénomènes et même en établir des éphémérides. Mais les forces qui entrent en jeu produisent dans les deux cas des déformations et engendrent des tensions dans tout le globe. Ces déformations à leur tour additionnant leur effet à celui du potentiel initial modifient les caractéristiques des phénomènes. En première approximation, comme il s'agit de phénomènes à longue période vis à vis de la période de vibration propre de la Terre $\left(\mathrm{I}^{\mathrm{h}} 34^{\mathrm{m}}\right)$, on peut considérer que ces effets obéissent à la théorie statique. Ce sont donc les amplitudes des phénomènes qui sont modifiées: réduites ou augmentées suivant les manifestations du phénomène que l'on considère.

Ainsi dans le cas du mouvement du pôle, le pôle d'inertie vrai tend à se rapprocher du pôle instantané de rotation et le suit dans son mouvement; comme toute observation astronomique 
du pôle d'inertie vrai est impossible, nous sommes amenés à prendre pour origine de référence un certain pôle d'inertie "moyen" que nous définissons un peu arbitrairement ( $c f$. Troisième Problème) comme le barycentre de la polhodie couvrant six années (commensurabilité approximative des deux périodes fondamentales reconnues dans le mouvement du pôle).

Cette manière inéluctable de procéder donne l'apparence d'un allongement de période - c'est l'allongement chandlérien-il n'est que l'image du déplacement du pôle d'inertie vers le pôle de rotation, déplacement dâ à l'action de la force centrifuge sur la matière élastique qui constitue la Terre et dont l'amplitude est fonction du module de rigidité de la planète. Tel est le lien existant entre la période de Chandler et les propriétés élastiques de la Terre.

On voit qu'il s'agit en somme d'introduire dans les calculs de la Mécanique Céleste les paramètres représentant l'état physique interne de la Terre. On avait dans le passé déjà tenté de discuter l'effet de la distribution radiale des densités (paramètre de Radau-aplatissement dynamique); On tente aujourd'hui d'introduire la distribution radiale du module de rigidité, le coefficient de viscosité, l'effet des convections dans le noyau, etc. . . . .

2. Examinons d'abord de plus près comment la théorie permet d'associer à ce phénomène astronomique le phénomène géophysique des marées terrestres. L'idée fondamentale de la théorie, due à Love, est que, le potentiel étant représenté (avec une approximation suffisante) par une fonction harmonique sphérique du second ordre, toutes les perturbations produites dans la Terre par ce potentiel peuvent être représentées par la même fonction harmonique, affectée d'un facteur numérique d'amplitude correspondant à l'aspect du phénomène particulier envisagé; ce facteur est appelé nombre de Love; pour certaines manifestations de la déformation ce sont des combinaisons algébriques simples des nombres de Love que l'on sera amené à introduire.

A titre d'exemple, le déplacement radial $U$, la dilatation cubique $D$ produits en tout point de la Terre par les forces dérivant d'un potentiel du second ordre $W_{2}$ sont donnés par

$$
\begin{aligned}
& U=H(r) W_{2} / g \\
& D=f(r) W_{2} / g,
\end{aligned}
$$

$r$ étant la distance du centre de la Terre au point considéré. Par voie de conséquence le potentiel dû à la déformation et à la variation de densité provenant de la dilatation cubique sera donné également par une expression de la forme

$$
V=K(r) W_{2}
$$

tandis que les déformations dans le méridien et le premier vertical s'écriront

$$
L(r) \frac{\delta W_{2}}{g \delta \theta} \quad L(r) \frac{\delta W_{2}}{g \sin \theta \delta \lambda} .
$$

Le potentiel $W_{2}$ peut être aussi bien le potentiel d'attraction luni-solaire que le potentiel de la force centrifuge provenant du mouvement de l'axe de rotation par rapport à la Terre:

$W_{2}=V-V_{0}=\frac{1}{2} \omega^{2} r^{2}\left(\sin ^{2} \phi^{\prime}-\sin ^{2} \phi\right)$

$$
\text { où } \phi^{\prime}=\phi-x \cos \lambda-y \sin \lambda \text {, }
$$

$(x y)$ coordonnées du Service International des Latitudes, $\lambda=$ longitude.

A la surface de la Terre $(r=a)$ on pose

$$
H(a)=h \quad K(a)=k \quad L(a)=l
$$

qui sont les nombres de Love usuels:

$h=$ le rapport de la hauteur de la marée terrestre à la hauteur de la marée statique océanique correspondante,

$k=$ le rapport du potentiel additionnel dû à cette déformation au potentiel déformateur lui-même.

L'amplitude des déviations de la verticale par rapport à la croûte, dues aux marées, est affectée du coefficient $\gamma=\mathrm{I}+k-h$, les déviations de la verticale par rapport à l'axe du monde du coefficient $\Lambda=\mathbf{I}+k-l$, les variations de l'intensité de la pesanteur, du coefficient $\delta=\mathrm{I}+h$ $-\frac{3}{2} k$. On a trouvé $\gamma=0,706 ; \delta=\mathbf{I}, 20$; $\Lambda=\mathbf{I}, \mathbf{1} 5$, d'où $h=0,482$ et $k=0,188$.

Pour fixer les idées sur les valeurs numériques de ces nombres, notons que:

$$
\begin{array}{clll}
\mathrm{I}^{\circ} \begin{array}{c}
\text { pour une Terre } \\
\text { fluide homogène }
\end{array} & h=\mathrm{I} & k=0,6 & l=0,3 \\
2^{\circ} \text { pour une Terre in- } & h=0 & k=0 & l=0 \\
\text { finiment rigide } & & & \\
3^{\circ} \text { pour la Terre réelle } & h=0,482 & k=0,188 & l=0,06
\end{array}
$$

Les nombres $h, k, l$ sont évidemment liés à la distribution radiale des constantes élastiques et de la densité du milieu par des équations différentielles (Herglotz) complexes (généralisation de l'équation de Clairaut) dont l'intégration numérique a été exécutée pour la première fois par Takeuchi et ensuite par Molodensky. Toute une série de modèles de la constitution interne du globe ont ainsi été éprouvés et certains donnent des résultats assez voisins de l'expérience bien 
qu'un accord parfait entre les nombres $\gamma$ et $\delta$ ne soit pas encore obtenu. L'introduction de la graine dans les calculs le permettra sans doute.

On montrera maintenant comment la valeur numérique du nombre $k$ détermine la valeur de la période du mouvement libre du pôle instantané de rotation à la surface de la Terre, dite période de Chandler.

Le potentiel perturbateur $W_{2}$ dâ au mouvement du pôle (force centrifuge) peut être représenté par une fonction harmonique tesserale du second ordre, tout comme le potentiel des marées diurnes (les marées semi-diurnes et à longue période étant représentées par des fonctions harmoniques du second ordre respectivement sectorielles et zonales).

La déformation consécutive à l'action de ce potentiel $W_{2}$ et qui lui est proportionnelle induit un nouveau potentiel additionnel $k W_{2}$ et introduit en même temps de petits produits d'inertie non nuls dans les équations d'Euler. Ces produits d'inertie contiennent donc en facteur le nombre $k$ et peuvent aisément être exprimés en fonction de la différence des moments principaux d'inertie polaire et équatorial $C-A$ :

$$
\begin{aligned}
& D=-k y \mu(C-A) \\
& E=-k x \mu(C-A) \\
& F=0
\end{aligned}
$$

avec

$$
\frac{\mu}{\mathrm{I}+\mu}=\frac{\mathbf{I}}{\epsilon} \frac{\omega^{2} a}{2 g},
$$

où $(x y)$ sont les coordonnées du Service International des Latitudes,

$\omega=$ vitesse angulaire de rotation de la Terre,

$a=$ rayon de la Terre,

$g=$ intensité de la pesanteur,

$\epsilon=$ aplatissement de la Terre.

Les équations du mouvement s'écrivent alors

$$
\begin{aligned}
& \frac{d x}{d t}+\frac{C-A}{A}(\mathrm{I}-k \mu) \omega y=0, \\
& \frac{d y}{d t}-\frac{C-A}{A}(\mathrm{I}-k \mu) \omega x=0,
\end{aligned}
$$

et la période du mouvement libre est

$$
\tau=\frac{2 \pi}{\omega} \frac{A}{C-A} \frac{\mathrm{I}}{\mathrm{I}-k \mu},
$$

relation qui est indépendante de toute hypothèse concernant la structure interne de la Terre, sauf dans la définition et la valeur de $k$.

On voit aussitôt que pour $k=0$, cas de la
Terre rigide, on retrouve la période classique d'Euler:

$$
\tau_{0}=\frac{2 \pi}{\omega} \frac{A}{C-A}=305 \text { jours sidéraux. }
$$

On a donc

$$
k=\frac{\mathrm{I}}{\mu}\left(\mathrm{I}-\frac{\tau_{0}}{\tau}\right) .
$$

La valeur très sûre donnée pour $k$ par la discussion des observations de marées terrestres $(k=0,188)$ conduirait à une période de Chandler de 382 jours, en net désaccord avec tous les résultats d'analyse harmonique effectués sur les courbes décrites depuis 70 ans par le pôle.

Notons toutefois que l'on devrait apporter une correction à cette période pour tenir compte de la contribution des océans dans les produits d'inertie de déformation. On arriverait ainsi à une valeur maximum de $k$ d'environ 0,23 soit à une période de 400 jours: le désaccord avec l'observation est à peine atténué.

3. L'analyse harmonique de toutes les données récoltées par le Service International des Latitudes depuis sa fondation fournit en effet la valeur moyenne de 429,5 jours pour la période de Chandler (Melchior). Cette analyse harmonique a pu être menée de bien des manières mais les résultats ne sont pas significativement différents.

Cependant, si l'on admet à priori que le mouvement obéit à une certaine loi analytique et si l'on tient compte de la forme de cette loi pour exécuter le calcul, on peut être conduit à des résultats qui ne sont pas nécessairement en accord avec ceux des analyses exécutées sans schéma préconçu. C'est ce qu'ont fait Walker et Young en admettant, après Dyson et Jeffreys que le mouvement est amorti dans les conditions établies par le postulat de l'élastico-viscosité de Maxwell. Dans ce cas les équations s'écrivent sous la forme générale:

$$
x_{i}+\lambda x_{l}+\chi x_{j}=0 .
$$

En appliquant le schéma d'autorégression de Yule, Walker et Young parviennent à une période de 440 jours (désaccord encore plus prononcé avec les marées terrestres) et un temps de relaxation de 3 ans. $\tau=\frac{2 \pi}{\lambda}=$ la durée après laquelle l'amplitude $\alpha$ est réduite à $e^{-1} \alpha$ soit 0,37 de l'amplitude initiale.

Gutenberg a mis en doute ce résultat, montrant que si l'on admet pour le module de rigidité les valeurs déduites des vitesses de propagation 
des ondes sismiques confrontées avec l'amplitude des marées terrestres et en admettant pour le coefficient de viscosité la valeur déduite de la vitesse du relèvement finno-scandien (Niskanen), on arriverait d̀ un temps de relaxation de plusieurs centaines d'années.

Aucune explication n'a d'autre part pu être trouvée pour un amortissement aussi fort. Melchior a mis en doute sa signification en montrant que les formules de Yule reposaient sur le postulat de Maxwell, lequel n'a aucune base physique: on peut donc douter de la validité de ces équations adoptées pour représenter le mouvement du pôle et penser que les valeurs numériques trouvées par Walker et Young sont des paramètres donnant une bonne représentation mathématique mais dénués de sens physique.

Cependant une difficulté supplémentaire réside dans le fait que la période du mouvement du pôle apparaît comme nettement variable et qu'aucune démonstration théorique n'a encore pu être donnée pour expliquer un tel phénomène.

4. Un inconvénient majeur réside dans le fait que la suite des coordonnées $(x y)$ du pôle manque d'homogénéité pour des raisons diverses que nous énumérons brièvement:

$I^{\circ} \mathrm{La}$ nécessité d'assurer dans chaque groupe d'étoiles observé une compensation aussi parfaite que possible des mesures micrométriques (de sorte que les erreurs inévitables sur la valeur admise pour les pas de vis ne se répercutent pas sur les latitudes déduites des observations) oblige à des révisions périodiques du programme d'étoiles à observer: substitutions de certaines étoiles ou paires d'étoiles rétablissant un équilibre que la précession tend à détruire systématiquement au cours du temps. Ceci fut fait en 1906, I9I2, I922, I935, I954. Mais l'effet de ces changements de catalogue fut plus sensible encore lorsque les systèmes auxquels ils étaient référés ont également été changés: AGKI en I900, G. C. Boss depuis 1935 .

$2^{\circ} \mathrm{La}$ méthode de réduction a varié selon les conceptions des savants qui furent chargés de la réduction d'ensemble des données. Les variantes successives ont évidemment toutes été introduites dans un but de perfectionnement. Mais leur juxtaposition fait que le procédé actuellement en usage diffère sensiblement de celui qui fut mis en oeuvre au début du siècle par Albrecht. Les anciennes séries d'observations devraient donc être revues selon les critères actuellement admis.

A titre d'exemples, on introduit maintenant de petits termes de nutation (Ross) les termes d'aberration de Battermann, tandis que les composantes périodiques locales empiriques de Kimura ont été abandonnées. Par contre l'interprétation du terme $z$ de Kimura et de l'erreur de fermeture est à présent bien assise. On peut logiquement considérer le terme de Kimura comme dû essentiellement aux erreurs de déclinaisons du type $\Delta \delta_{\alpha}$ avec en surplus de petites contributions dues aux erreurs commises sur les valeurs admises pour les constantes fondamentales de nutation et d'aberration.

L'erreur de fermeture est pour 60 pour-cent due à l'erreur sur la constante d'aberration (laquelle devrait être portée à 20 ". $5 \mathrm{I}$ ); pour le reste elle paraît bien liée aux variations de la pression atmosphérique au cours de la nuit (Hattori). Il est bien évident que des effets météorologiques locaux assez difficiles à définir jouent un rôle non négligeable dans ces deux caractéristiques des observations tandis que la nature de l'instrument, son type de construction ne joue pas de rôle apparent (sauf pour le télescope flottant de Cookson, qui est assez sensible aux effets du vent, ce qui n'est guère surprenant).

Ainsi donc une nouvelle réduction d'ensemble doit avant tout se baser sur un catalogue homogène établi dans le système du $\mathrm{FK}_{4}$ (la construction de ce catalogue est en cours à l'Observatoire Royal de Belgique). Elle doit être faite sur la base d'une méthode de réduction soigneusement mise au point, méthode qui ne différerait pas sensiblement de celle du Professeur Cecchini, sauf considération aux variations à longue période des latitudes locales qui devraient être éliminées selon les vues d'Orlov mais avec une combinaison d'ordonnées plus sélective que la sienne ( $c f$. III.I).

Dans cette réduction, on devra adopter la valeur 20 ". 5 I pour la constante d'aberration et une valeur améliorée de la constante de nutation. Enfin, l'effet des marées terrestres sur la direction de la verticale locale par rapport à l'axe du monde doit être pris en considération puisque l'opération inverse a permis de déduire la marée des observations même de latitude du Service International et d'en donner une définition des nombres de Love qui est très cohérente avec les observations purement géophysiques ( $c f$. I.6).

Nous pensons que ce n'est qu'après un tel travail-à vrai dire très long et très délicat-que l'on pourra avoir la certitude que les caractéristiques des composantes du mouvement du pôle 
sont absolument indépendantes et du procédé d'observation et du procédé de réduction.

5. Considérons maintenant les fluctuations de la vitesse de rotation de la Terre autour de son axe instantané de rotation. Parmi ces fluctuations il existe des composantes qui résultent des déformations de la Terre provoquées par des marées terrestres du type zonal (et qui sont les marées dites à longue période). Les déformations zonales modifient en effet le plus grand moment d'inertie $C$ du globe et l'on peut s'attendre par conséquent à une variation périodique correspondante de la vitesse de rotation de la Terre en vertu de la formule:

$$
\frac{d \omega}{\omega}=-\frac{d C}{C} .
$$

Ainsi l'on retrouvera l'effet de la marée solaire semi-annuelle $S s a$ et des marées lunaires mensuelle $M m(27,56$ jours $)$ et semi-mensuelle $M f$ (13,66 jours) dans les fluctuations de la vitesse de rotation. Elles ont été mises en évidence pour la première fois par W. Markowitz dans les observations au P.Z.T. L'amplitude des marées lunaires est de l'ordre de la milliseconde et contient en facteur le nombre $k$. En effet, le potentiel $k W_{2}$ engendré par la déformation zonale considérée s'exprime en fonction des variations du moment d'inertie principal $C$.

L'amplitude de la composante périodique de la variation de vitesse est donc bien liée au nombre $k$ et par conséquent l'observation astronomique de haute précision est appelée à fournir bientôt une nouvelle valeur expérimentale de ce nombre.

Cette méthode nous est très précieuse en raison de l'impasse dans laquelle on se trouve actuellement par suite du désaccord entre la valeur "chandlérienne" de $k$ et la valeur déduite des observations purement géophysiques de marées terrestres.

En ce qui concerne la marée solaire semi-annuelle $S s a, N$. Stoyko avait déjà montré en I950 qu'elle permettait d'expliquer la plus grande partie de la variation semi-annuelle de la vitesse de rotation. Les résultats de Markowitz, indiquant une amplitude de ro millisecondes, le confirment.

6. Enfin, les méthodes astronomiques mises en oeuvre pour l'étude de la rotation de la Terre constituent encore deux méthodes indépendantes pour la détermination expérimentale des nombres de Love. On sait, en effet, que les déviations de la verticale sont observées par les géophysi- ciens à l'aide de pendules horizontaux très sensibles placés à grande profondeur (de l'ordre de Ioo mètres). Cette manière d'observer les déviations de la verticale n'offre d'autre référence que la croûte mobile elle-même et introduit en facteur la combinaison $\gamma=\mathbf{I}+k-h$ des nombres de Love. Il est évident que ces déviations périodiques de la verticale affectent les déterminations de latitude de haute précision et que de très longues séries d'observations telles que celles du Service International des Latitudes (ou de certains observatoires s'étant consacrés à ce problème) sont susceptibles de les mettre en évidence. Dans ce cas cependant les observations ne se réfèrent plus à la croûte mais à l'axe du monde et le coefficient d'amplitude est la combinaison $\Lambda=\mathbf{I}+k-l$. La discussion de tout l'ensemble de données actuellement disponible a permis de fixer pour ce coefficient la valeur

$$
\Lambda=\mathbf{I}+k-l=\mathbf{I}, \mathbf{I} 5
$$

qui est en excellent accord avec les mesures géophysiques.

Le second procédé astronomique de détermination expérimentale des nombres de Love résulte de la comparaison d'horloges à balancier de haute précision avec des horloges à quartz. Les horloges à balancier sont abandonnés pour la conservation de l'heure mais leur marche présentait cependant cet intérêt d'être liée à la valeur instantanée de $g$, laquelle varie en fonction de l'attraction luni-solaire et de la deformation du globe qui en résulte; les horloges à quartz en. sont indépendantes. Le facteur d'amplitude rendant compte de l'effet de la déformation élastique est ici, comme pour les gravimètres, la combinaison $\delta=\mathrm{I}+h-\frac{3}{2} k$. Une étude très soigneuse due à N. Stoyko et portant sur quatre années et cinq pendules de l'Observatoire de Paris a donné pour résultat

$$
\delta=\mathbf{I}, 2 \mathrm{I}
$$

en accord parfait avec les résultats gravimétriques: $\delta=\mathrm{I}, 2 \mathrm{O}$.

II

I. L'existence d'une composante annuelle importante aussi bien dans les fluctuations de la vitesse de rotation que dans le mouvement du pôle est évidemment liée aux déplacements de masse à période annuelle à la surface de la Terre : mouvements de masses d'air et d'eau obéissant au cycle saisonnier (c'est donc un effet solaire indirect). 
2. L'importance de cet effet dans le mouvement du pôle s'explique par l'existence d'une résonance avec la période propre (période de Chandler).

Les équations du mouvement du pôle s'écrivent $\frac{d}{d t}(x+i y)-i \chi(x+i y)=-i \chi(\xi+i \eta)$,

où $(x y)$ sont les coordonnées du pôle instantané de rotation publiees par le S.I.L., $(\xi \eta)$ les coordonnées du pôle instantané d'inertie, $\tau=2 \pi / \chi$, période de Chandler, $T=2 \pi / \alpha$, période annuelle. On les intègre aisément en admettant le mouvement du pôle d'inertie elliptique (le calcul d'après les données météorologiques montre qu'il est quasi linéaire) :

et l'on a

$$
\xi+i \eta=n e^{i \alpha t}+n^{\prime} e^{-i \alpha t}
$$

$$
x+i y=R e^{i \chi t}-m e^{i \alpha t}+m^{\prime} e^{-i \alpha t} .
$$

$R e^{i x t}$ représente le mouvement libre chandlérien (circulaire) tandis que les axes du mouvement elliptique annuel du pôle instantané de rotation sont donnés par

$$
\begin{aligned}
& b=m^{\prime}-m=\frac{n^{\prime}}{\tau+\mathrm{I}}-\frac{n}{\tau-\mathrm{I}} \\
& a=m^{\prime}+m=\frac{n^{\prime}}{\tau+\mathrm{I}}+\frac{n}{\tau-\mathrm{I}}
\end{aligned}
$$

cubiques en $(a, \tau)$ et $(b, \tau)$ qui sont très bien satisfaites par les résultats de l'analyse harmonique des mouvements du pôle (I900-1955) et qui, compte tenu des variations observées de la période $\tau$, conduisent pour les axes de l'ellipse décrite par le pôle d'inertie aux valeurs:

$$
A=n+n^{\prime}=\text { o". } 012, \quad B=n-n^{\prime}=0 \text { ". } 040
$$

en très bon accord avec les calculs géophysiques de Rosenhead (calcul de l'effet des déplacements de masses d'air et d'eau, compte tenu d'une compensation due à la flexion de la croûte terrestre):

$$
A=\text { o".or I } B=\text { o".o44. }
$$

3. Ce raisonnement suppose que l'amortissement du mouvement libre n'est pas sensible à l'échelle de la précision actuelle, c'est à dire que le temps de relaxation serait supérieur à 20 ans. Mais en admettant avec Walker et Young un temps de relaxation de trois ans, la résonance agissant sur le mouvement forcé annuel est fortement réduite et si l'on prend aussi pour la période de Chandler la valeur de 440 jours, l'amplitude du mouvement du pôle d'inertie devrait être multipliée par un facteur trois pour rendre compte de la composante annuelle observée dans le mouvement du pôle de rotation.

Les axes de l'ellipse représentant le mouvement du pôle d'inertie devraient donc avoir pour longueur

$$
A=0 \text { o.036 } B=0 \text { o". } 120
$$

ce qui ne s'accorde plus avec les calculs de Rosenhead.

Cependant Munk et Groves ont pris en considération l'effet des vents qui se manifeste par un échange de quantités de mouvement et une pression sur les chaînes de montagnes (l'Himalaya, orienté selon un parallèle, jouant là un rôle essentiel); ils ont obtenu:

$$
A=\text { o".o I } \quad B=\text { o". I I4 }
$$

mais l'incertitude qui règne sur leurs données de base et précisément sur l'effet dans l'Himalaya qu'ils ont traité d'une manière hypothétique rend ces valeurs peu sûres.

Par contre si l'on admet que l'amortissement n'est pas sensible $(\tau \geqslant 20$ ans), et que les variations observées dans la période de Chandler sont réelles (bien qu'inexpliquées), il devient aisé d'expliquer les variations d'amplitude correspondantes de la composante annuelle comme une conséquence de la variation de l'effet de résonance.

Ceci montre en tous cas que le problème n'est pas résolu et que les résultats météorologiques de l'A.G.I. permettront certainement de le traiter avec plus de sécurité.

Notons encore que si le mouvement subissait un tel amortissement, il faudrait encore trouver la source de son entretien; on n'a pu la trouver ni dans les phénomènes atmosphériques irréguliers ni dans le noyau liquide.

Quoiqu'il en soit notre conclusion est que la précision actuelle (1900-I958) des déterminations de la position du pôle ne permet pas une détermination du temps de relaxation si celui-ci est supérieur à 20 ans, ce qui paraît bien être le cas.

4. Les considérations qui précèdent évoquent immédiatement l'existence d'une corrélation avec la fluctuation annuelle de la vitesse de rotation de le Terre. Cependant dans ce cas les déplacements de masses ne jouent qu'un faible rôle et ce sont surtout les vents qui, par des échanges de quantités de mouvement, expliquent le phénomène.

Le léger retard par rapport à la phase des saisons astronomiques n'est pas inattendu, 
TABLEAU I. LES CORRELATIONS AVEC LA FLUCTATION ANNUELLE

(PHASE $t$ EXPRIMÉE EN FRACTIONS D'ANNÉES)

Vitesse de rotation*
Accélération

Elongation du pôle d'inertie†

Elongation du pôle de rotation

Milieu des saisons

astronomiques

* D'après N. Stoyko.

† D'après P. Melchior.

\begin{tabular}{|c|c|c|}
\hline $\begin{array}{c}\underset{\text { ninimum }}{\operatorname{mulle}} \\
0,104\end{array}$ & $\begin{array}{c}\text { moyenne } \\
\text { maximum }(+) \\
0,354\end{array}$ & $\begin{array}{c}\text { maximum } \\
\text { nulle } \\
0,604\end{array}$ \\
\hline $\begin{array}{l}\underset{0,102}{\operatorname{minimum}} \\
\text { maximum }\end{array}$ & $\begin{array}{l}\underset{0,357}{\operatorname{maximum}} \\
\text { minimum }\end{array}$ & $\begin{array}{l}\underset{0,612}{\operatorname{minim} u m} \\
\text { maximum }\end{array}$ \\
\hline $\begin{array}{l}\text { hiver } \\
0,096\end{array}$ & $\begin{array}{c}\text { printemps } \\
0,342\end{array}$ & $\begin{array}{c}\text { été } \\
0,597\end{array}$ \\
\hline
\end{tabular}

$$
\begin{gathered}
\text { moyenne } \\
\text { maximum }(-) \\
0,854 \\
\text { maximum } \\
\text { minimum } \\
0,854 \\
\text { automne } \\
0,84 \mathbf{I}
\end{gathered}
$$

Phase moyenne 0,4790

0,4812

0,4702 puisque les phénomènes considérés sont dus à une accumulation ou à une dissipation d'énergie par l'atmosphère et les océans.

\section{III}

I. A ces phénomènes annuels, chandlériens et luni-solaires, se superpose aussi bien dans le cas du mouvement du pôle que dans le cas des fluctuations de la vitesse de rotation de la Terre un effet dit séculaire en ce sens qu'on n'y relève pas de périodicité définie soit parce que nos observations ne s'étendent pas suffisamment loin dans les siècles passés, soit parce qu'il n'en existe pas.

Ces dérives ne sont pas linéaires, elles présentent des inversions, des perturbations, des discontinuités. Comme dans les deux cas précédents on a tenté naturellement d'établir une corrélation entre les accidents qui ont affecté la vitesse de rotation et ceux qui ont affecté la position du pôle.

2. La question d'une dérive séculaire possible du pôle est d'une très grande importance en géologie et en géophysique, mais pour l'instant, nous ne pouvons nous baser que sur à peine plus d'un demi-siècle d'observations réalisées par le Service International des Latitudes. Les observations antérieures ne sont ni de la même précision ni de la même homogénéité; en outre elles sont basées sur des catalogues d'étoiles de précision nettement insuffisante. Même dans le cas du Service des Latitudes, l'homogénéité est loin d'être parfaite et les discontinuités dont nous avons parlé plus haut (I.4) empêchent, comme nous le montrerons plus loin, de tirer actuellement une conclusion tant soit peu sûre sur cette dérive hypothétique. Le problème du mouvement séculaire est étroitement lié à la définition exacte de la position du pôle d'inertie moyen, de la latitude moyenne de chaque station et de la stabilité de cette latitude.
3. Le problème de la détermination de la latitude moyenne des stations du Service des Latitudes est un problème controversé du point de vue du procédé de réduction à adopter pour les observations de latitude. Il sera nécessaire de trancher la question au moment où l'on se proposera d'exécuter la révision d'ensemble de toutes les observations faites depuis I900. On ne peut, en effet, prétendre que les stations de latitude soient douées d'une stabilité parfaite. Certaines d'entre elles présentent même des glissements relativement importants (Mizusawa, Tschardjui). Toutes les stations, comme l'a montré A. J. Orlov, présentent en outre de lentes fluctuations dans leur latitude moyenne, fluctuations dont l'origine n'a pas été définie mais d'où un mouvement réel du pôle doit être exclu, car on constate qu'elles sont différentes pour des stations situées sur le même méridien ou même pour deux instruments opérant en une même station. Il faudra donc éliminer ces fluctuations de la latitude moyenne du calcul de la polhodie, sous réserve de les discuter séparément.

Or, tous les calculs du Service des Latitudes sont effectués au millième de seconde, la latitude moyenne devra donc être donnée pour chaque dixième année avec cette précision par une combinaison adéquate des données encadrant ce dixième d'année (méthode de Labrouste). Orlov a proposé que l'on élimine ces effets locaux des variations de latitude avant le calcul des coordonnées du pôle en définissant à tout instant la latitude moyenne par la combinaison linéaire d'ordonnées,

$$
\phi_{m}=\frac{\mathrm{I}}{2 \mathrm{O}} \sum_{t=0}^{4}\left(\phi_{t}+\phi_{t+5}+\phi_{t+6}+\phi_{t+11}\right) .
$$

Il est évident qu'on ne pourra admettre pour les réductions des latitudes moyennes présentant d'une dixième d'année à l'autre des variations à caractère brusque ou accidentel (même dans le millième de seconde), c'est à dire que la courbe 
représentant les fluctuations de la latitued moyenne dont on tiendra compte doit être très adoucie.

L'application de la méthode d'Orlov montre que cette combinaison d'ordonnées ne filtre pas rigoureusement les courtes périodes. Or tel devait être l'objet de l'opération. La combinaison d'Orlov a été construite, en effet, de manière à présenter, dans sa courbe de sélectivité un zéro pour $n=$ Io, période annuelle, et un zéro pour $n=\mathrm{I} 2$, période de Chandler, ( $n$ étant compté en dixièmes d'années). Or ceci n'assure pas l'élimination rigoureuse des effets dûs au mouvement du pôle car les périodes des composantes de celui-ci ne sont pas définies avec cette sûreté, en particulier la période libre.

On est en présence, à cet égard, de deux hypothèses: ou bien l'on a affaire à une période libre oscillant entre les valeurs $n=\mathrm{I}$ I,3 et $n=\mathrm{I} 2, \mathrm{o}$ ou bien l'on a affaire à un spectre de bandes correspondant à la résonance du système mécanique excité par des fluctuations désordonnées (d'origine non précisée) ce spectre de bandes est assez large. Pour éliminer ces composantes, il importe peu de fixer exactement les zéros de la courbe de sélectivité à $n=10$ et $n=12$; il est plutôt nécessaire de choisir une combinaison complexe d'addition qui possède une série de zéros bien répartis entre $n=3$ et $n=15$, de manière à filtrer rigoureusement toutes les courtes périodes. Du point de vue purement théorique on peut donc critiquer la méthode d'Orlov parce qu'elle ne répond pas exactement aux caractéristiques physiques du mouvement du pôle. En outre, sa sélectivité n'est pas suffisante pour assurer l'élimination des courtes périodes (définies par $n \leq 15$ ).

Pour choisir une méthode plus sélective, nous nous sommes donné pour conditions de centrer la combinaison sur un dixième d'anneé exact et d'éliminer toutes les périodes inférieures à $n=$ I5. La combinaison complexe de rang supérieur à introduire sera telle que $n=15 \geqslant 2 m+\mathrm{I}$ soit $m \leq 7$; cette combinaison sera $s_{7}$. Nous avons porté notre choix sur la combinaison $s_{4} s_{5} s_{6} s_{7}$ (notations de Labrouste).

Le facteur d'amplification pour les longues périodes est 20 dans la combinaison d'Orlov et dans la combinaison $s_{4} s_{5} s_{6} s_{7}, 99 \times 195=19,305$. Les courbes de sélectivité montrent évidemment la supériorité de la combinaison $s_{4} S_{5} s_{6} s_{7}$ sur celle d'Orlov.

En particulier la faible oscillation de période $T=0,7$ an, qu'introduit la combinaison d'Orlov dans les variations de latitude et qui correspond au maximum que présente la courbe de sélectivité pour cette période, est évidemment inexistante avec la combinaison $s_{4} s_{5} s_{6} s_{7}$.

4. Ceci étant posé, considérons la manière dont le mouvement séculaire du pôle "moyen" a pu être mis en évidence. Sekiguchi a opéré des moyennes glissantes sur six années sur les coordonnées du Service des Latitudes $x, y$; il a dû cependant corriger a priori les coordonnées publiées des discontinuités qui ont été introduites dans le système de référence à chaque changement de catalogue.

A la date d'un changement de catalogue ( $c f$. I.4), se produisent en effet à la fois une discontinuité et un changement de direction et de vitesse de la dérive. La discontinuité se traduit par une translation du système d'axes de coordonnées qui résulte en fait d'une variation inégale des latitudes moyennes des diverses stations; ces latitudes moyennes sont calculées par le Bureau Central sur chaque intervalle correspondant à un même catalogue.

Sekiguchi a utilisé des transformations de coordonnées calculées par Kimura et Nicolini d'après les changements apportés aux latitudes moyennes adoptées pour chaque station. La validité de ces transformations a été critiquée avec de bonnes raisons par Cecchini (déplacement réel de stations, telle Mizusawa). Pour déterminer sans hypothèse préalable et les dérives et les translations du système d'axes Melchior a appliqué aux coordonnées originales $(x y)$, sans aucune correction préalable et séparément sur chaque intervalle homogène, la combinaison d'ordonnées $s_{4} s_{5} s_{6} s_{7}$ dont les propriétés ont été décrites plus haut. Les dérives trouvées sont généralement linéaires et l'on peut se permettre de les extrapoler jusqu'aux frontières de chaque intervalle considéré. La discontinuité aux frontières donne la transformation de coordonnées au moment du changement de programme par une voie tout à fait indépendante de la précédente et l'on constate que les formules de Kimura-Nicolini sont valables. Ceci implique que les stations, telle Mizusawa ne se déplacent pas réellement et qu'il s'agit de dérives au sens d'Orlov, c'est à dire fictives et dépourvues de sens astronomique ou géophysique.

Ces dérives en $x$ et en $y$ sont absolument différentes d'un programme à l'autre. En outre Phillippov a démontré que les longues séries d'observations de Greenwich, Poulkovo et Washington sont mieux représentées par une polhodie sans dérive que par la polhodie avec dérive. Nous pensons donc qu'on ne peut rien conclure pour 
l'instant au sujet de la dérive du pôle moyen. Comme l'a remarqué avec pertinence Fedorov, si nous sommes forcés d'abandonner pour l'instant toute tentative d'étudier le mouvement séculaire du pôle, c'est évidemment moins grave que de parvenir à des conclusions fausses sur ce mouvement.

Ainsi donc le mouvement séculaire devrait être dissocié de celui de la polhodie, ce qui présenterait l'avantage de la centrer sur l'origine et de fournir des corrections de variations de longitude plus pratiques pour les observations horaires. Celà revient à éliminer les fluctuations de la latitude moyenne des diverses stations (comme nous en avons déjà montré la nécessité en III.3) et les arguments qui s'opposent à l'introduction de stations indépendantes du S.I.L. dans le calcul de la polhodie elle-même (et qui reposent essentiellement sur la nature du terme annuel $z$ de Kimura) ne jouent pas dans le cas de l'étude du mouvement séculaire à condition que toutes les courtes périodes et notamment celle d'un an soient correctement filtrées. Le mouvement séculaire doit être calculé à partir de toutes les stations où les variations de latitude ont été étudiées assez longtemps que pour permettre l'application de la combinaison $s_{4} s_{5} s_{6} s_{7}$. Les variations de longitudes éventuellement décelées par les opérations mondiales des longitudes pourraient également être introduites dans ces calculs.

5. Quelles peuvent être les causes de ces dérives? Constatant l'inexactitude des mouvements propres adoptés pour certaines étoiles des programmes et démontrant surtout que dans l'application du programme il existait une dissymétrie systématique marquée entre les diverses stations (fréquence variable d'observation d'étoiles faibles et d'étoiles en fin de groupe selon les observatoires). Melchior avait interprété la dérive comme dûe aux inexactitudes des mouvements propres des étoiles utilisées. Sur la base de cette interprétation Phillippov et Sekiguchi ont repris indépendamment les calculs de réduction et ont montré que la dérive subsistait indépendamment de cet effet.
Sans doute doit-on considérer que des effets atmosphériques interviennent ici mais d'une manière qui reste confuse pour nous (anomalies de réfraction liées à la dispostion des groupes d'étoiles par rapport au zénith?).

6. Ces restrictions étant faites sur l'exactitude des dérives que l'on peut mettre en évidence dans le mouvement du pôle, on considérera avec prudence les coincidences à vrai dire troublantes que l'on a relevées avec les variations séculaires de la vitesse de rotation de la Terre. Sekiguchi a remarqué qu'aux deux perturbations les plus marquées de la dérive du pôle moyen: 1922 et I936 étaient associées des perturbations aussi brusques de la vitesse de rotation de la Terre. Il propose de les attribuer à un couplage électromagnétique entre le noyau et l'écorce terrestre.

Enfin, faisant abstraction de ces perturbations et cherchant à déceler seulement l'allure moyenne de la dérive du pôle de I900 à I950, on constate avec Cecchini que le déplacement du centre de gravité de la polhodie (pôle d'inertie Nord). en 50 ans aurait été de 0 ".o9I ( 2,7 mètres) suivant le méridien $37^{\circ} 5 \mathrm{o}^{\prime} \mathrm{W}$ (direction de Terre Neuve) ce qui serait assez bien expliqué par la fonte des banquises et des glaciers et la répartition des eaux sur les océans.

\section{CONCLUSIONS}

Nous pensons avoir montré ici la complexité des problèmes qui restent à traiter. Le gain en précision que l'on obtiendra grâce aux nouveaux instruments conduira certes à de nouveaux progrès mais seule une très longue suite d'observations de tous ces phénomènes permettra d'en dégager les lois fondamentales.

\section{BIBLIOGRAPHIE SOMMAIRE}

(On trouvera une bibliographie detaillée dans ces ouvrages)

Melchior, P. 1954, Obs. Roy. Belgique Monographie, $\mathrm{n}^{\circ} 4$ - 1955, Comm. Obs. Roy. Belgique, no 79; 1958, Ibid, $\mathrm{n}^{\circ} \mathrm{I} 34$.

1957, "Latitude Variation" Progress in Physics and Chemistry of the Earth, Pergamon Press; Comm. Obs. Roy. Belgique, $\mathrm{n}^{\circ}$ 130).

1957, "Rapport sur les marées terrestres" (Assemblée Gen. U.G.G.I., Toronto).

\section{DISCUSSION}

Dr. PAuChenko* remarked that Dr. Melchior said in his communication that until now no one has given any explanation of very strong damping of the free nutation of the earth, as found

* Gravimetrical Observatory of the Academy of Sciences of the Ukrainian S.S.R., Poltava, U.S.S.R. by Drs. A. M. Walker and Andrew Young. These results may be questioned, not because of the defects in the method of analysis, but because of the character of the data used.

The method of harmonic analysis proposed by Dr. Jeffreys and developed by Drs. Walker and 